\title{
The quest for young asteroid families: new families, new results
}

\author{
V. Carruba, ${ }^{\star}$ E. R. De Oliveira, B. Rodrigues and I. Requena \\ School of Natural Sciences and Engineering, São Paulo State University (UNESP), Guaratinguetá, SP, 12516-410, Brazil
}

Accepted 2018 July 4. Received 2018 May 22; in original form 2018 February 12

\begin{abstract}
Asteroid families form as a result of collisions. The fragments resulting from the familyforming event are ejected into orbits near that of the parent body, and then start dynamically migrating because of gravitational and non-gravitational effects, such as the Yarkovsky force. Families that formed less than $20 \mathrm{Myr}$ ago are special, since their secular angles, the longitudes of pericenter and nodes, may still converge with respect to those of the putative parent body when integrated backward in time, at the moment of family formation. This allows for obtaining age estimates and family membership with a precision not allowable for other, more evolved asteroid families. This method of family dating, the Backward Integration Method, or BIM, has been, so far, successfully applied to the case of eight asteroid families. In the last years, however, because of the astounding rate of new asteroid discoveries, several new small and compact asteroid families have been identified. In this work, we apply the BIM to 28 asteroid families not previously studied with this method. We identified four families for which we observe a possible convergence of the angles. For three of them, we obtained age estimates: at a 68.3 per cent confidence level, (3152) Jones should be $1.9_{-1.9}^{+4.3}$, (7353) Kazuya should be $2.2_{-2.2}^{+1.4}$, and (108138) $2001 \mathrm{~GB}_{11}$ should be $4.6_{-1.1}^{+1.6}$ Myr old. (909) Ulla might be younger than $\simeq 6 \mathrm{Myr}$.
\end{abstract}

Key words: Celestial mechanics - Minor planets, asteroids: general-Minor planets, asteroids: individual: (3152) Jones, (7353) Kazuya, (108138) 2001 GB$_{11}$, (909) Ulla.

\section{INTRODUCTION}

Asteroid families form after a collisionary event shatters a parent body. The fragments formed after the collision are ejected into nearby orbits. Members of asteroid families are identifiable in domains of proper elements or frequencies. After the family forming event, gravitational and non-gravitational effects, such as the Yarkovsky force, can change the asteroid's proper elements and slowly disperse the family. The current orbital position of family members is, therefore, the product of the initial ejection velocity with which the fragment was expelled and of the subsequent dynamical evolution. Since the Yarkovsky force depends on asteroid size and physical parameters that are sometimes poorly known, such as the asteroid density and surface thermal conductivity, disentangling the two components that affected the asteroid position is not always easy. Monte Carlo methods that simulate the dynamical evolution and the effect of the original ejection velocity field can provide estimates of family ages, but those are affected by the uncertainties by which the key parameters controlling the strength of the Yarkovsky force are known.

For very young families, those that formed before $\simeq 20 \mathrm{Myr}$ ago (Nesvorný \& Bottke 2004; Radović 2017), an alternative approach

^E-mail: valerio.carruba@unesp.br to family dating is, however, possible. The differences of the longitudes of pericenter $\varpi$ and node $\Omega$ of family members with respect to those of the alleged parent body still converge in the past for these families at the time of family formation, allowing to estimate these families ages with a precision not available for other groups. The Backward Integrations Method (BIM, hereafter), first hypothesized by Brouwer, but successfully applied for the first time to a family by Nesvorný et al. (2002), has been an important tool for studying young asteroid families for more than 15 years. Yet, the number of families for which a solution of the convergence of secular angles has been found is still limited. So far, to our knowledge, only eight asteroid families have been dated with this approach.

In the last three years, however, the number of asteroids and asteroid families increased dramatically. Several new asteroid families and clumps have been identified by Milani et al. $(2014,2016)$ and Nesvorný, Brož \& Carruba (2015), among others. These includes several new, compact groups, many of which are possibly the results of cratering event (Milani et al. 2014, 2016), that have never before been studied with BIM. In this work, we will take advantage of the newly available list of family members and investigate if some of these new groups can be dated using this approach. We will start our work by first reviewing the current state-of-the-art of the results obtained with BIM. 


\section{BIM: STATE OF THE ART}

Results of the BIM were already discussed in Nesvorný et al. (2015) (see Table 1), and, more recently, in Radović (2017). Here, we briefly summarize the successful applications of this method that we are aware of. While the method of convergence of secular angles was originally proposed by Brouwer in the 1950s, the first actual application of the method to the Karin sub-family of the Koronis family was carried out by Nesvorný et al. (2002). Nesvorný \& Bottke (2004) developed methods to account for the past Yarkovskyinduced semi-major axis mobility of the same family, and estimated values of the changes in semi-major axis caused by this effect for the then known 97 members of the Karin family. More recently, Carruba, Nesvorný \& Vokrouhlický (2016) used a sample of family members about seven times larger to refine Nesvorný \& Bottke (2004) age estimate and to observe, for the first time, traces of YORP-induced mobility among the smallest members of the family.

The second family for which BIM was successfully applied was that of Veritas. Nesvorný et al. (2003) found an age estimate based on the convergence of $\Delta \Omega$ angles of $8.2 \pm 0.3$ Myr. Convergence in $\varpi$ was not found at the time, because of the proximity of this family to the 2/1 mean-motion resonance with Jupiter, that caused fast precessions of longitudes of pericenters. Recently, however, Carruba, Vokrouhlický \& Nesvorný (2017) refined the age estimate of Nesvorný et al. (2003) and obtained convergence in $\Delta \varpi$ for non-chaotic population of Veritas members using digitally filtered secular angles.

Nesvorný et al. (2003) also studied the case of the Nele family, then named Iannini, and, based on the non-uniform distribution of $\Omega$ and $\varpi$, suggested a young age for this family. This result is confirmed by new results that show that the Nele family should be younger than 7 Myr (Carruba et al. 2018). Nesvorný et al. (2008) identified the case of the Beagle group, a sub-family of the larger Themis family. Past convergence of nodal longitudes suggested an age of less than 10 Myr for this group, but older ages could not be excluded. As for the case of the Veritas family, the proximity to the $2 / 1$ resonance with Jupiter caused fast precessions of $\varpi$ for this family as well.

After 2008, the research group of Dr. Novaković used BIM to date four asteroid families, that of Theobalda(Novaković 2010), the interesting case of Lorre (Novaković, Hsieh \& Cellino 2012a), a C-type family in a dynamically stable environment, identified also using single- and multi-oppositions asteroid orbits, and two active asteroid families, those of (300163) P/2006 VW139 (Novaković et al. 2012b), and P/2012 F5 (Gibbs) (Novaković et al. 2014).

Finally, there was also the cases of families that formed less than 1 Myr ago, many of which are so young that can only be identified in osculating or mean element domains (Nesvorný \& Vokrouhlický 2006; Nesvorný, Vokrouhlický \& Bottke 2006; Vokrouhlický \& Nesvorný 2011; Pravec et al. 2018). Results for 12 of these clusters are reported in the last rows of Table 1. We used for the age estimates of these groups the latest available results from Pravec et al. (2018).

Excluding Koronis 2, a family that was first identified by Molnar \& Haegert (2009) and that is supposed to be quite young (Brož et al. 2013), and Beagle (Nesvorný et al. 2008), for both of which a good solution of secular angles convergence is not yet available, there are just eight families older than $1 \mathrm{Myr}$ and identifiable in proper element domains for which a good age estimate has been found so far with BIM: Karin, Veritas, Nele, Theobalda, Lorre, (300163) P/2006 VW139, and P/2012 F5 (Gibbs), with the last two being families around active asteroids in the main belt. Table 1 displays a list of the results obtained with BIM so far. The FIN, family identification number, was a number introduced by Nesvorný et al. (2015) to track the history of each family. ${ }^{1} C_{0}$ was a parameter used by Nesvorný et al. (2015) to date asteroid families. Smaller values of $C_{0}$ are associated with younger ages. In the next section, we will discuss the cases of newly identified asteroid families.

\section{RECENTLY IDENTIFIED ASTEROID FA MILIES}

Several new asteroid families have been identified in the last few years Milani et al. (2014); Nesvorný et al. 2015; Milani et al. 2016, and many of them have never been investigated, to our knowledge, with the BIM. In this work, we focus on the new families listed in Nesvorný et al. (2015). Other, more updated families lists are currently available, for instance at the AstDyS2 website: http://hamilton.dm.unipi.it/astdys/index.php?pc=5 (Milani et al. 2014, 2016). In this exploratory work, however, we prefer to focus on the Nesvorný et al. (2015) list because i) it provides preliminary information on family ages of some new, compact families, and ii) we wanted to have an unique, coherent system, rather than study the results of different families classifications obtained at different times, so as to be able to discern possible patterns associated to the families for which BIM is successful. Families not identified by Nesvorný et al. (2015) or not present in our list remains worthy challenges for future lines of research.

To identify which new families could be good candidates to be studied with BIM, we used these selection criteria: i) for families listed in Nesvorný et al. (2015) and for which a $C_{0}$ value is available, since the higher $C_{0}$ values observed for a family analysed with BIM was 0.20 for Veritas, we selected families with $C_{0}<0.20$. In the other cases, we selected families defined as ii) compact in Nesvorný et al. (2015), or iii) with fewer than 100 members in the same source. Both these types of families have the potential to be the product of a recent collisionary event. Table 2 lists the relevant data for the families that passed this phase of selection. Overall, we found 28 families never before investigated with the BIM.

Next, we applied BIM to these families. We integrated all family members, as listed in Nesvorný et al. (2015) backward in time, over the influence of all planets and a time-step of one day, for $21 \mathrm{Myr}$, long enough to sample the time for which BIM can be successfully applied. We used $S W I F T \_M V S F$, which is a symplectic integrator from Levison \& Duncan (1994). The code was modified by Brož (1999) to include an online filtering of the osculating elements. The Yarkovsky effect was not included in this initial integration. Using the largest body in each family as a reference orbit, we computed values of $\Delta \varpi=\varpi_{a s t}-\varpi_{\text {ref }}$ and $\Delta \Omega=\Omega_{a s t}-\Omega_{\text {ref }}$ at each time. We then computed a $\chi^{2}$-like variable of the form:

$\chi_{0}^{2}=\sum_{i=2}^{N_{\text {ast }}}\left(\Delta{\varpi_{i}}^{2}+\Delta \Omega_{i}{ }^{2}\right)$,

where $N_{\text {ast }}$ is the number of family members. At the optimal family age, the difference between the $\Delta \varpi_{i}$ and $\Delta \Omega_{i}$ should be minimal, and so should be the value of $\Delta \chi_{0}^{2}=\chi_{0}^{2}-\chi_{0 \text { min }}^{2}$, where

\footnotetext{
${ }^{1}$ Since there are cases in which the lowest numbered asteroid in a family later on turned out to be an interloper, with a subsequent change of name of the family, by assigning a FIN to each family, Nesvorný et al. (2015) introduced a tool that could allow different researchers to easily track each family's identification history.
} 
Table 1. A list of families for which the ages were determined using the backward integration method. The table reports the family identification number of Nesvorný et al. (2015), the family identification, the estimated age, values of the $C_{0}$ parameter of Nesvorný et al. (2015), when available, and some references and notes. The last 12 families are families mostly younger than $1 \mathrm{Myr}$, that were identified in domains of osculating or mean orbital elements. The ages estimates reported in the table for these clusters are the latest available results from Pravec et al. (2018). For clusters for which multiple formations events are possible, we report the ages of each event, as determined by Pravec et al. (2018).

\begin{tabular}{|c|c|c|c|c|}
\hline FIN & $\begin{array}{l}\text { Family } \\
\text { Name }\end{array}$ & $\begin{array}{l}\text { Estimated } \\
\text { age [Myr] }\end{array}$ & $\begin{array}{c}C_{0} \\
{\left[10^{-4} \mathrm{au}\right]}\end{array}$ & $\begin{array}{l}\text { References and } \\
\text { Notes }\end{array}$ \\
\hline 610 & (832) Karin & $5.75 \pm 0.05$ & $0.03 \pm 0.01$ & Nesvorný et al. (2002); Nesvorný \& Bottke (2004); Carruba et al. (2016) \\
\hline 520 & (1547) Nele & $<7$ & - & Nesvorný et al. (2003); Carruba et al. (2018), chaotic dynamics \\
\hline 617 & (778) Theobalda & $6.9 \pm 2.3$ & - & 778 offset, Novaković (2010) \\
\hline 640 & P/2012 F5 (Gibbs) & $1.5 \pm 0.1$ & - & Novaković et al. (2014), main belt comet \\
\hline 621 & (158) Koronis(2) & $15 \pm 5$ & $0.010 \pm 0.005$ & Molnar \& Haegert (2009), age from Brož et al. (2013), difficult angles conv. \\
\hline 620 & (656) Beagle & $<10 ?$ & $0.07 \pm 0.03$ & Nesvorný et al. (2008), possibly older than $10 \mathrm{Myr}$ \\
\hline 412 & (21509) Lucascavin & $0.37_{-0.10}^{+0.64}, 0.88_{-0.37}^{+0.27}$ & - & Nesvorný \& Vokrouhlický (2006), 3 members, multiple disruptions? \\
\hline 523 & (14627) Emilkowalski & $0.30_{-0.07}^{+0.04}, 1.16_{-0.16}^{+0.35}$ & - & Nesvorný \& Vokrouhlický (2006), 6 members, multiple disruptions? \\
\hline 524 & (16598) Brugmansia & $0.17 \pm 0.06$ & - & Nesvorný \& Vokrouhlický (2006), 3 members \\
\hline- & (18777) Hobson & $0.365 \pm 0.067$ & - & Pravec \& Vokrouhlický (2009); Rosaev \& Plávalová (2017), 11 members \\
\hline- & (22280) Mandragora & $0.29 \pm 0.20$ & - & Pravec et al. (2018), 18 members \\
\hline- & (39991) Iochroma & $0.14_{-0.07}^{+0.13}$ & - & Pravec \& Vokrouhlický (2009), 5 members \\
\hline- & (66583) Nicandra & $0.89_{-0.06}^{+0.21}$ & - & Pravec et al. (2018), 5 members \\
\hline
\end{tabular}

$\chi_{0 \min }^{2}$ is the lowest value of the $\chi_{0}^{2}$ variable. At this stage of our investigation, we do not try to assess the probability confidence level of our solution, but we purely determine the qualitative behaviour of the $\chi_{0}^{2}$-like variable. The goodness of the solutions found with this approach for the best cases will be better investigated in the next section, where we will also include the Yarkovsky effect in our modelling.

Three main types of behaviour for the $\chi_{0}^{2}$ variable are possible and are shown in Fig. 1. In panel (a) of Fig. 1 we show a case where BIM works well, and there is a single, well-defined minimum of the $\chi_{0}^{2}$ function. Panel (b) show a less conclusive case, in which there are more than one minimum over the studied time interval, while panel (c) displays a case in which the family is probably older than $21 \mathrm{Myr}$, since $\chi_{0}^{2}$ values are consistently very high over the whole length of the integration, with only isolated spikes of $\Delta \chi_{0}^{2}$.

We applied this type of analysis to the 28 families of Table 2, and our results for families of the first two types are summarized in the sixth column of Table 2. Most of the analysed families are type $\mathrm{C}$ and cannot be dated with BIM. However, we found two families for which BIM seems to have found an unique age solution, those of (7353) Kazuya and (3152) Jones, and four cases in which there might be an age solution (types B of fig. 1): those of (108138) $2001 \mathrm{~GB}_{11}$, (918) Itha, (89) Julia, and (909) Ulla. The convergence of secular angles for the A- and B-type families when the Yarkovsky force is considered will be treated in the next section.

\section{PAST CONVERGENCE OF THE LONGITUDES WITH NON-GRAVITATIONAL FORCES}

To refine the convergence in both $\Delta \varpi$ and $\Delta \Omega$, we performed numerical simulations accounting for the Yarkovsky drifts of the considered asteroid families. For each member of the type A and B families, we created 71 clones with identical initial orbits. These clones were then integrated with $S W I F T_{-} R M V S 3_{-} D A$, a symplectic integrator based on $S W I F T_{-} R M V S 3$ code (Levison \& Duncan 1994) that was modified by Nesvorný \& Bottke (2004) to include a constant drift in the semi-major axis, over $21 \mathrm{Myr}$ and with a time step of 10 days. Each clone was assigned a different drift, covering plus or minus the maximum possible drift. For the maximum drift values, we use the typical values for S-type (3.5 $\left.10^{-10} \mathrm{au} / \mathrm{d}\right)$ and C-type $\left(5.510^{-10} \mathrm{au} / \mathrm{d}\right) 1 \mathrm{~km}$ diameter objects, as from Spoto, Milani \& Knežević (2015), multiplied by a factor 1.5. The last factor was motivated by the fact that there are uncertainties by which asteroid densities and thermal conductivities are known, and those can modify the drift rates by factors of a order 2 or more. ${ }^{2}$ Also, it has been shown (Delbo et al. 2007; Vokrouhlický et al. 2015) that large asteroids can retain larger grains on their surface, and, therefore, their thermal conductivity may be higher, affecting the Yarkovsky drift rate. Asteroid families with no known types, like that of (108138) $2001 \mathrm{~GB}_{11}$, were assigned the larger C-type drift by default. Drift rates for objects at diameters different than 1 were obtained by assuming an inverse dependence of drift rates with respect to the asteroid size. Asteroid sizes were estimated using absolute magnitudes and the mean values of geometric albedos, as

\footnotetext{
${ }^{2}$ The choice of the factor 1.5 is also motivated by the fact that using too large drift rates may cause the smallest family members to drift too fast, causing them to reach orbits with significantly different values of proper frequencies with respect to the parent body over the length of the integration, so losing information of the time of convergence of secular angles on shorter timescales. Experience with the case of the Nele family (Carruba et al. 2018) showed that the factor 1.5 appears to be an optimal trade-off for studies of families with BIM.
} 
Table 2. Possible young asteroid families, as listed by Nesvorný et al. (2015). The FIN, family name, cutoff distance $\left(d_{c u t}\right)$ at which the family was identified by HCM in Nesvorný et al. (2015), number of family members, values of the $C_{0}$ parameters, and some reference and notes for the asteroid families listed in Nesvorný et al. (2015) that passed this phase of selection are listed in column 1,2,3,4, and 5, respectively. The last column reports the type of temporal behavior of the $\chi_{0}^{2}$ function defined in equation (1) according to the classification shown in Fig. 1.

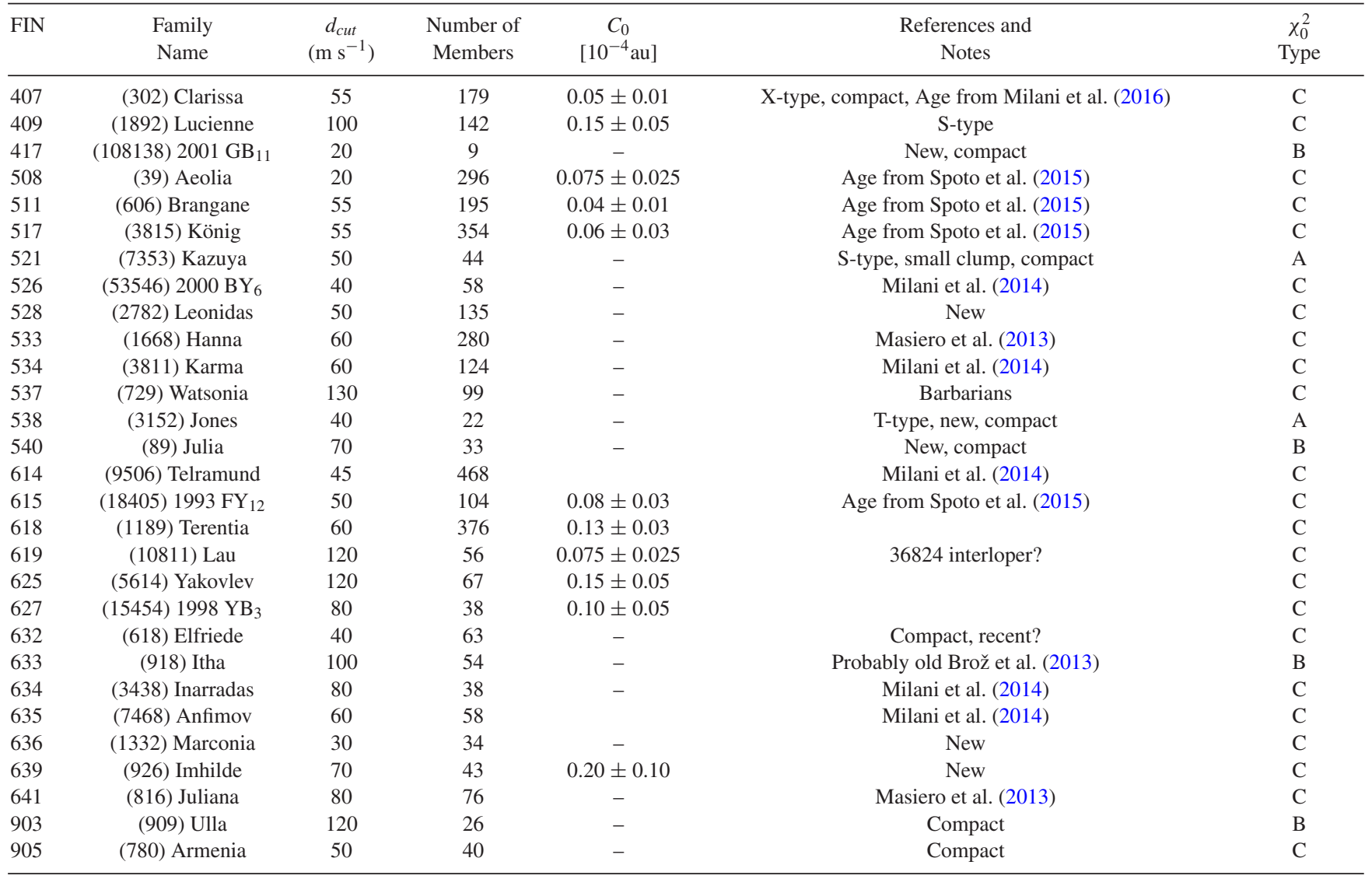

available from Spoto et al. (2015); Nesvorný et al. (2015). Finally, out of the 71 clones of each particle, we selected the clone that showed the best convergence with respect to the reference orbit at each time step.

As a first step in our analysis, we looked at the time behaviour of the $\Delta \chi_{0}^{2}$ data obtained for these new simulations, and checked for the presence of a well-defined minimum. Unfortunately, at times greater than $\simeq 7 \mathrm{Myr}$, the algorithm for the detection of the $\Delta \chi_{0}^{2}$ will pick whatever clone is closer to the reference orbit at that time. Because of the fast precession of the secular angles after this time, it will be always possible to have one out of the 71 clones close enough at that given time with respect to the reference orbit. Minima of $\Delta \chi_{0}^{2}$ found at times greater than $7 \mathrm{Myr}$ should therefore be considered as an artefact of this procedure. This, regrettably, further reduces the time-span for which BIM can be successfully applied below the $\simeq 20$ Myr limit for which Nesvorný \& Bottke (2004); Radović (2017) showed that no information can be obtained on the family age using BIM. Out of the six studied families, two did not displayed a minimum in the behaviour of $\Delta \chi_{0}^{2}$ over the first $\simeq 7 \mathrm{Myr}$, those of (89) Julia and (918) Itha (see Fig. 2). While an age older than $7 \mathrm{Myr}$ cannot be excluded for these two families based on this numerical experiment, we are unable at the current stage of this analysis to obtain a reliable age estimate for these two groups. (918) Itha, however, was estimated to be an old family by Brož et al. (2013).

The remaining four other studied families, however, displayed at least one local minimum over the first $7 \mathrm{Myr}$ of the integration. In order to obtain an age estimate for these cases, we adopted the following procedure: first, we computed values of $\chi_{0}^{2}$ with equation (1), and obtained its minimum value $\left(\chi_{0}^{2}\right)_{\min }$. To obtain a better age estimate, and eliminate short-period change in the osculating elements, this time both differences in angles were filtered with a low-pass digital Fourier filter. This procedure helped to remove all frequency terms with periods shorter than $10^{5} \mathrm{yr}$ (Carruba 2010). We tried other reasonable period thresholds in our filtering procedure, but all resulted in very similar conclusions. In order to estimate the errors on the convergence of the angles $\Delta \varpi$ and $\Delta \Omega$, we assumed that the errors were equal for all angles differences and caused the minimum spread at the estimated time of family formation. With these assumptions, we have that:

$\sigma^{2}=\frac{\left(\chi_{0}^{2}\right)_{\min }}{N_{\mathrm{DF}}}$

where $N_{\mathrm{DF}}=N_{\text {ast }}-2$ is the number of degrees of freedom of the $\chi_{0}^{2}$-like variable, since there were $2\left(N_{\text {ast }}-1\right)$ number of pairs contributing by angular differences $\Delta \varpi_{i}$ and $\Delta \Omega_{i}$ with respect to the reference orbit, and $N_{\text {ast }}-1$ clones were selected at each time-step by our procedure. An additional factor 1 was caused by the fact that we are fitting for the family age. Based on this error analysis, we can then define a proper $\chi^{2}$ variable using the relationship

$\chi^{2}=\sum_{i=2}^{N_{\text {ast }}}\left[\left(\frac{\Delta{\varpi_{i}}^{2}}{\sigma^{2}}\right)+\left(\frac{\Delta \Omega_{i}{ }^{2}}{\sigma^{2}}\right)\right]$, 

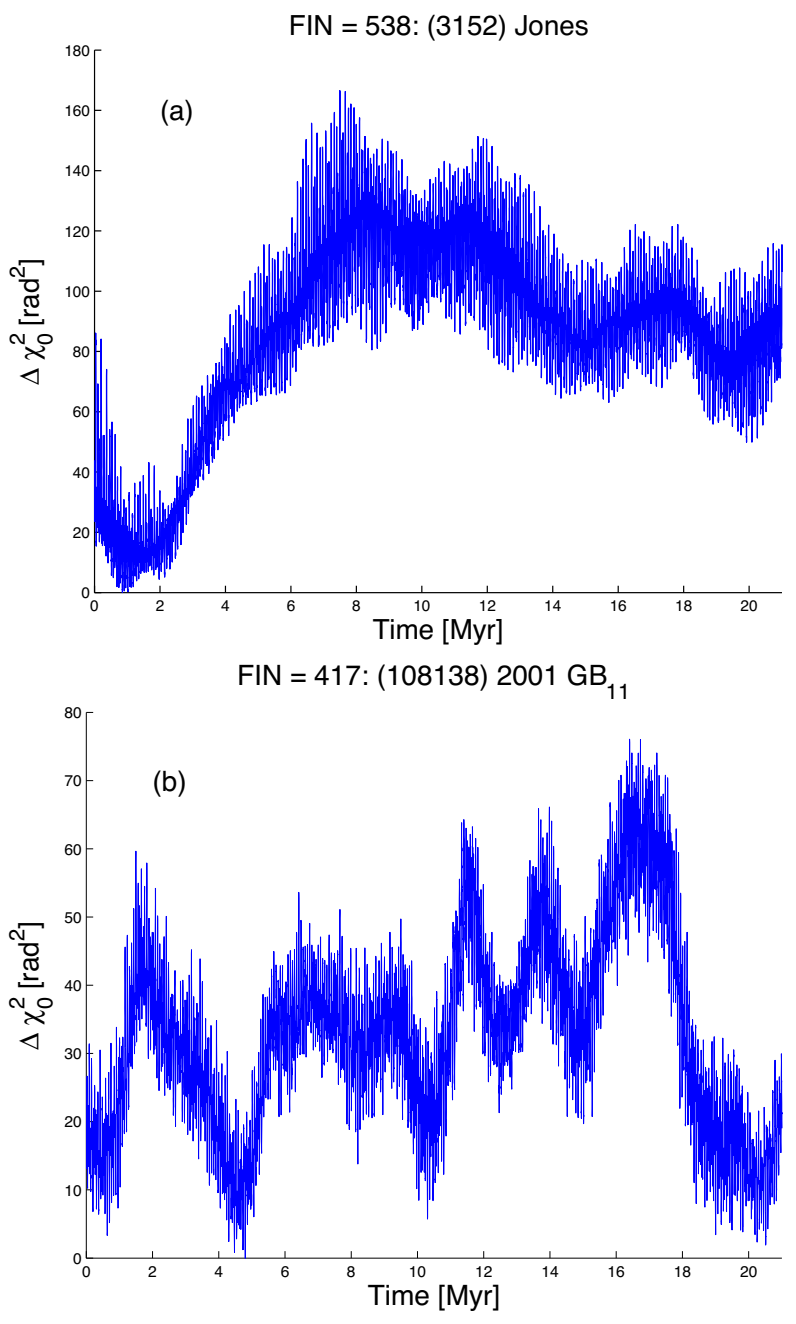

$\mathrm{FIN}=632(618)$ Elfriede

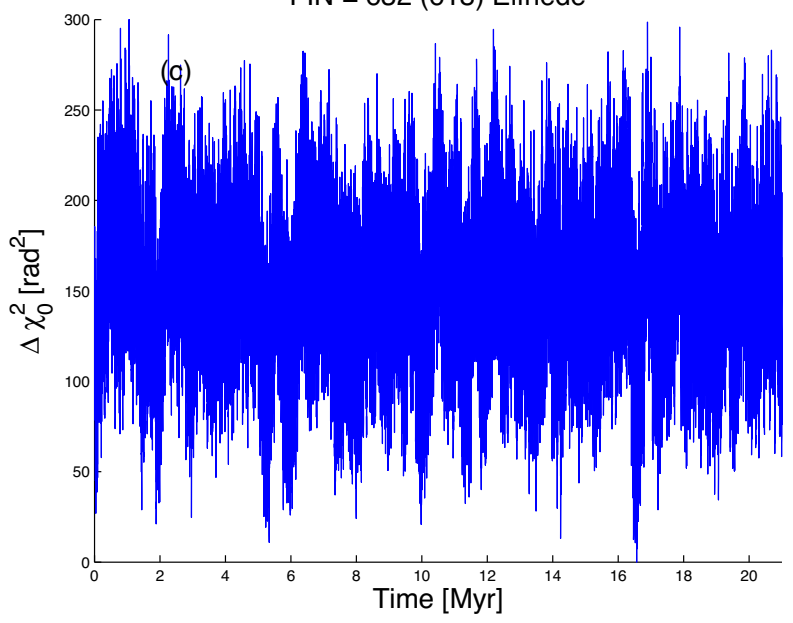

Figure 1. Three types of time behaviour for $\Delta \chi_{0}^{2}=\chi_{0}^{2}-\chi_{0 \min }^{2}$, with $\chi_{0}^{2}$ defined in equation (1). Panel (a) shows the results for a family with a single, well-defined minimum. Panel (b) those for a family with several minima, and panel (c) the case for which there is not a well-defined solution over the studied time range. Angles are expressed in radians. and then compute values of $\Delta \chi^{2}=\chi^{2}-\chi_{\min }^{2}$, where $\chi_{\min }^{2}$ is the new $\chi^{2}$ minimum. Probability confidence levels for the family age can then be estimated using the cumulative distribution function for a $\chi^{2}$ variable, computed for the appropriate number of degrees of freedom, estimated to be equal to $N_{\text {ast }}-2$.

Our results are shown in Fig. 3. The best case, among those studied, was that of the Jones family, shown in panel (a). For this family, we found a well-defined minimum 1.9 Myr ago, associated with a value of $\left(\chi_{0}^{2}\right)_{\min }=47.9 \mathrm{rad}^{2} .21$ out of the 22 members, 95.5 percent of the total, had their angles converging to within $\pm 80^{\circ}$ at the estimated family age. In panel (b) of Fig. 3 we show the values of the drift rates of the selected clones at the estimated time of family formation. The red curve shows the maximum and minimum drift rates, according to our convention. While there is a tendency for drift rates to cluster towards extreme values, we also observe intermediate drift rates, which suggest that those values should be reasonable. Results of this analysis are summarized in Table 3.

The second best case was that of the Kazuya family, the only S-type family among the four for which BIM was able to find a solution. Also, for this family, we found a well-defined and unique minimum, which, if we neglect the $\chi^{2}$ time evolution after $\simeq 4 \mathrm{Myr}$, is associated with a sole age estimate. However, in this case, only 22 out of 44 family members converged at the estimated family age, and the $\left(\chi_{0}^{2}\right)_{\min }$ value is also large ${ }^{3}$ More than for the Jones family, most drift rate values tend to cluster towards the extreme range (see panel (d)). This may suggest that a large portion of the Kazuya dynamical family may not actually be real family members, but interlopers. Further study of this group should be warranted before a definitive conclusion on this family age could be achieved.

(108138) $2001 \mathrm{~GB}_{11}$ (panel (e)) displayed a minimum at 4.6 Myr, associated with a $1-\sigma$ level probability of the family being $4.6_{-1.1}^{+1.6}$ Myr old. Eight out of nine members ( 88.9 per cent) had their angles converging to within $\pm 80^{\circ}$, and the drift rates were spread between the extreme values (panel (f)). However, this is a very small family of just nine members, and therefore the age estimate can be affected by small-number statistics issues.

Finally, the case of the (909) Ulla family was more problematic. Three members of the family are on unstable orbits and we could not find suitable clones of these objects for the duration of the simulation. Also, rather than a single well-defined $\Delta \chi_{0}^{2}$ minimum, there were several local minima in the first $8 \mathrm{Myr}$ of the data. Panel (g) displays our best solution if we assume that the minimum associated with the family forming event occurred $5 \mathrm{Myr}$ ago, which yields an age of $5.0_{-0.9}^{+0.4}$. Twenty-one out of 23 members had their secular angles converging at the estimated family age, and drift rates (panel (h)) did not cluster at extreme values. However, to within the errors, other solutions are also possible, and this age estimate is not well-defined. Further study is therefore needed to confirm or deny the preliminary estimates obtained with this method.

\footnotetext{
${ }^{3}$ The number of converging asteroids and the minimum of $\chi_{0}$ increase to 32 (72.7 percent of the total) and decrease to 76.6 if we use for Kazuya members values of drift rates typical of C-type asteroids. However, in this case, while the nominal age remain the same, $2.2 \mathrm{Myr}$, information on the convergence of secular angles is lost faster than for the case in which we used the smaller S-type drift rates, and we are not able to set an upper limit on the family age for this simulation.
} 

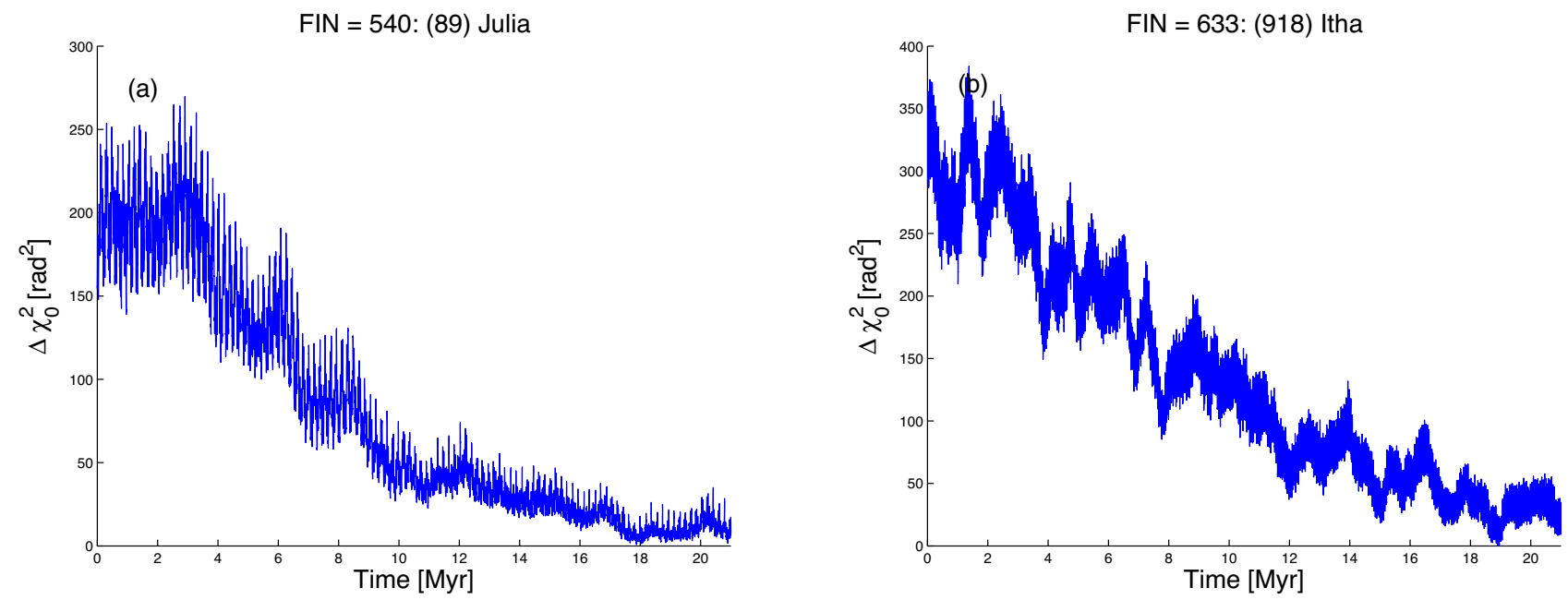

Figure 2. Time behavior of the $\Delta \chi_{0}^{2}$ variable for clones of members of the (89) Julia (panel (a)) and (918) Itha (panel (b)) families.

\section{CONCLUSIONS}

Our results could be summarized as follows:

(i) Revised the current state-of-the-art on the use of the Backward Integration Method (BIM) for obtaining age estimates of asteroid families. Currently, the method was used to obtain age estimates for 16 young asteroid families. As shown by Nesvorný \& Bottke (2004); Radović (2017), the method can only be applied to asteroid families that are $20 \mathrm{Myr}$ old or younger.

(ii) We selected 28 newly identified asteroid families (Milani et al. 2014; Nesvorný et al. 2015; Milani et al. 2016) never before analyzed with BIM, that were either considered young by Nesvorný et al. (2015), or were either compact and with fewer than 100 members according to the same source. We used BIM in a purely conservative scenario, without non-gravitational forces, and identified six families that could be younger than 20 Myr: the (7353) Kazuya, 3152) Jones, the (108138) $2001 \mathrm{~GB}_{11}$, (89) Julia, (918) Itha, and (909) Ulla families. A lower limit on the age of $\simeq 20$ Myr can be inferred for the other 22 studied asteroid families.

(iii) We integrated clones of members of the six candidate families when the Yarkovsky force was considered. At a 68.3 percent probability confidence lever,(3152) Jones should be $1.9_{-1.9}^{+4.3}$, (7353) Kazuya should be $2.2_{-2.2}^{+1.4}$, (108138) $2001 \mathrm{~GB}_{11}$ should be $4.6_{-1.1}^{+1.6}$ Myr old, and (909) Ulla might be younger than $\simeq 6$ Myr. Further study is however needed to confirm the age estimates obtained in this work. For instance, currently no information is available for any of the spin axis obliquities of the four family members in the DAMIT data base (Ďrech, Sidorin \& Kaasalainen 2010). If such information would become available for a significant fraction of these families members, and considering the enormous improvements in pole solutions for many main belt asteroids (Ďurech et al. 2015) this hypothesis does not seem outlandish, the precision with which the family age could be known will significantly improve. Also, the four asteroid families for which we obtained a preliminary age estimate were all classified as "compact" in Section 3, and, with the exception of (909) Ulla, which is located in the low number density region of the Cybele asteroids (Carruba et al. 2015), all were identified for values of $d_{\text {cut }}$ smaller than $50 \mathrm{~m} \mathrm{~s}^{-1}$. This suggests that, among the new families yet to be discovered, the ones classifiable as "compact" seems to be the most promising ones to be studied with BIM. To quantify how much a family is compact we computed for all the families and the clusters reported in this paper their standard deviation in proper $a, \sigma_{a}$. Fig. 4, panel (a) displays a histogram of this parameter for the family with a BIM solution and for those without. Excluding the peculiar case of (909) Ulla, all BIM families have values of $\sigma_{a}$ lower than $0.0075 \mathrm{au}$. This can be a useful quantity to check for future surveys of newly identified asteroid families, for which a BIM solution may seem likely.

We then also plotted the ages of all the families and clusters for which a solution with BIM is currently available versus $\sigma_{a}$ in Fig. 4, panel (b). In this Hertzsprung-Russell like diagram for young asteroid clusters, two main regions can be identified: the area in green is mostly populated by clusters of asteroids identified in domain of osculating or mean elements, while the area in yellow is the region where most of the families identified in domains of proper elements are currently found, and for which a reliable age that also account for the Yarkovsky drift rate can be found. (Nesvorný \& Vokrouhlický 2006) already suggested that there was a dichotomy between asteroid families found in domains of proper elements and the clusters found in domains of osculating elements in terms of dispersions in proper $(a, e, \sin i)$, with the latter being two order of magnitudes more compact. Current data on young asteroid families is, however, on the brink of closing the gap between the two regions. The asteroid family (20674), associated with the active asteroid P/2012 F5 (Gibbs), identified by Novaković et al. (2014) was also recently re-identified and studied by Pravec et al. (2018), which suggested the intriguing possibility of two events of fissions for the formation of this group. Three of the families identified as young with BIM in this work, those of (3152) Jones, (7353) Kazuya, and (108138) $2001 \mathrm{~GB}_{11}$, are also characterized by very small values of $\sigma_{a}$, and could also potentially be the product of a fission event rather than being the product of a collision, as proposed for most of the clusters in the green area (Jacobson \& Scheeres 2011; Vokrouhlický et al. 2017). Once again, future research will be needed to clarify the possible mechanisms of formation of these clusters.

Overall, we were able to identify four new asteroid families for which BIM could potentially provide information on ages and members drift rates. Each of these families deserves, in our opinion, to be studied in more detail than what we were able to achieve in this preliminary work. Several new objects could have been discovered since the works of Milani et al. (2014); Nesvorný et al. 2015; Milani et al. 2016, and this could potentially im- 

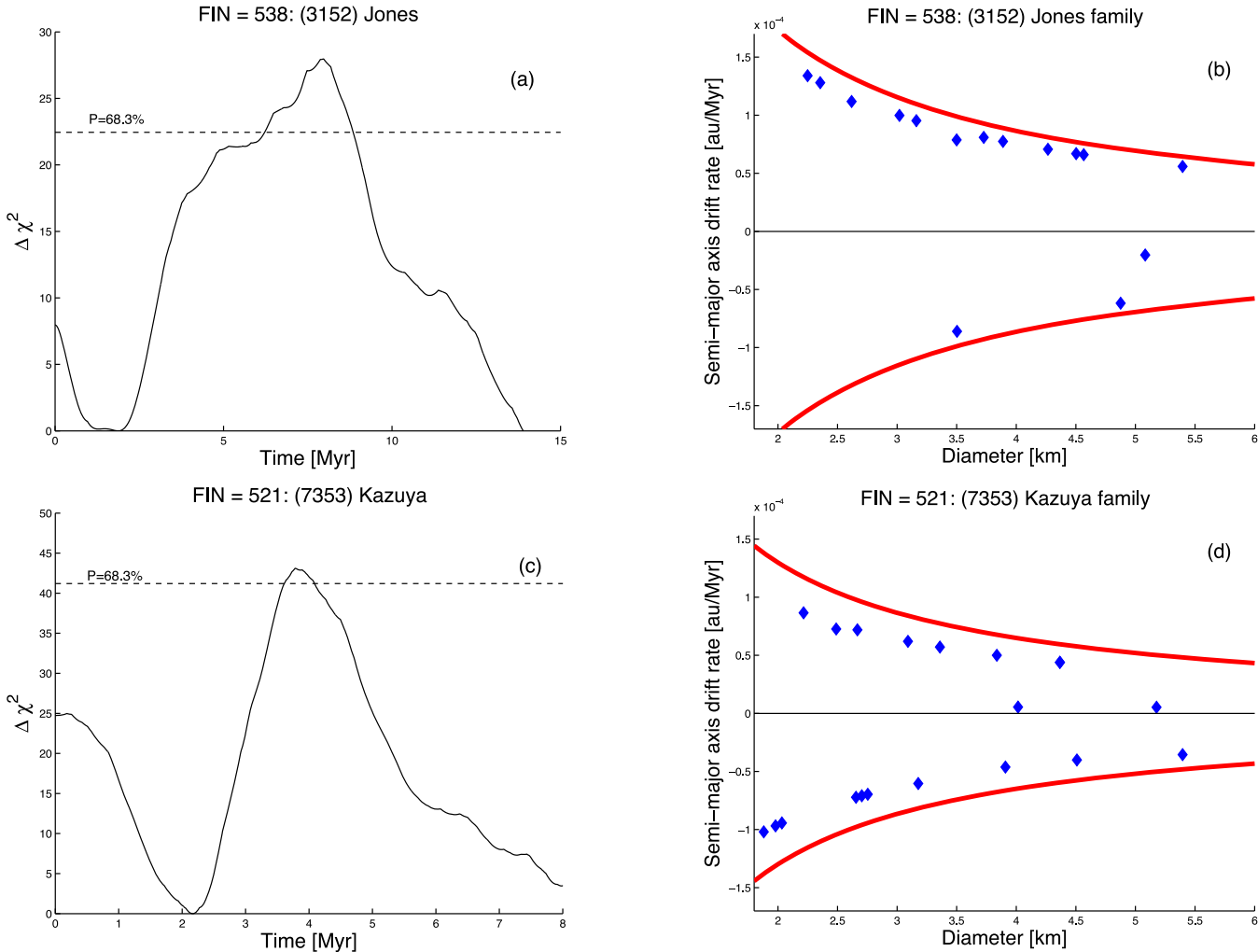

$\mathrm{FIN}=417:(108138) 2001 \mathrm{~GB}_{11}$
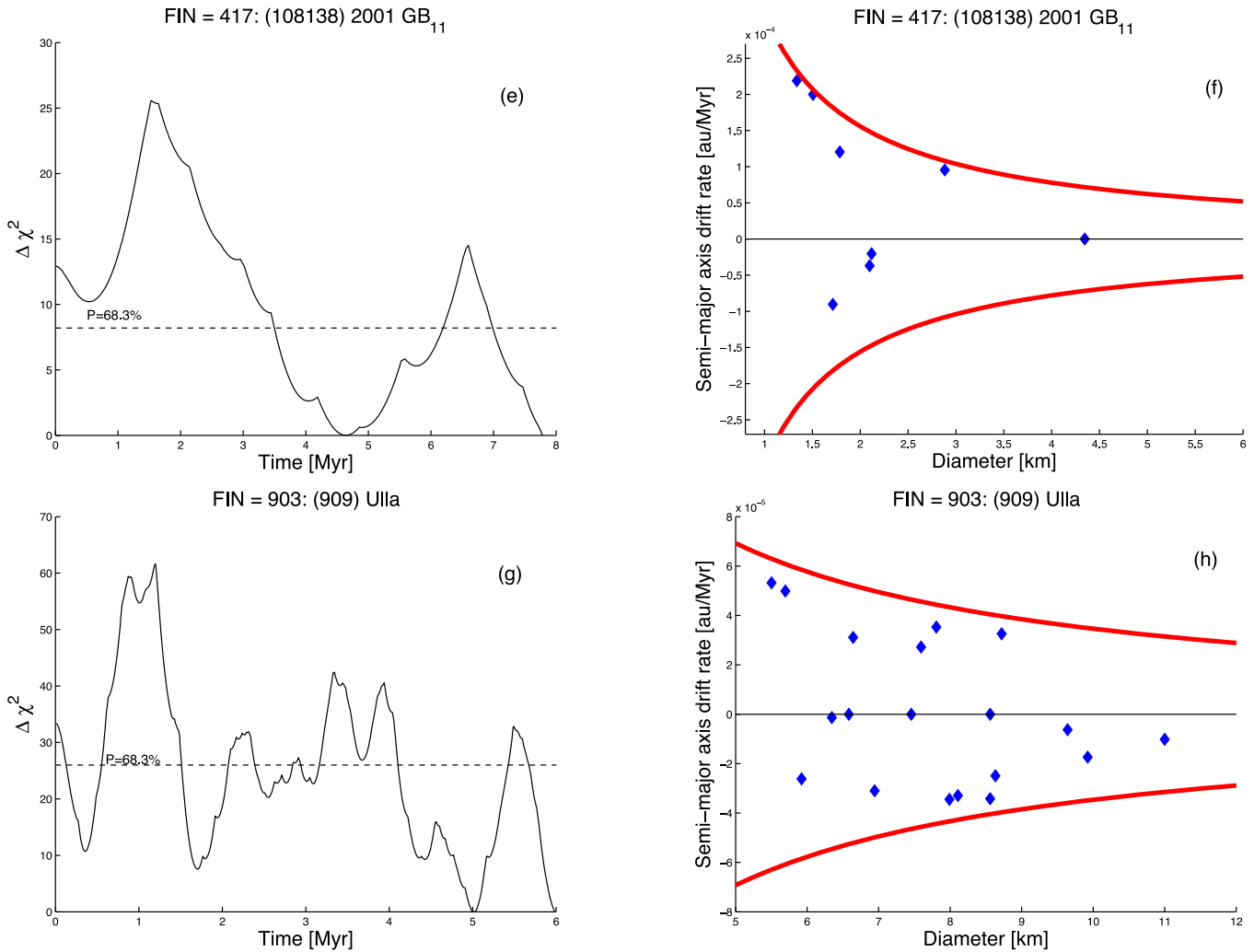

Figure 3. The left panels display the temporal evolution of the $\chi^{2}$ variable, as defined in equation (3) for clones of members of the (3152) Jones, (7353) Kazuya, (108138) $2001 \mathrm{~GB}_{11}$, and (909) Ulla families. The horizontal line shows the 68.3 per cent (1- $\sigma$ ) probability confidence level. The right-hand panels show the semi-major axis drift rate $\frac{d a}{d t}$ of family members. Red lines show the maximum $\frac{d a}{d t}$ values, according to Spoto et al. (2015) and our convention.

prove the $\chi^{2}$ statistics when the Yarkovsky effect is considered for the six studied families. More important, new young fami- lies could exist among the newly discovered bodies (see, for instance, the updated list available on the AstDyS-2 website: http: 

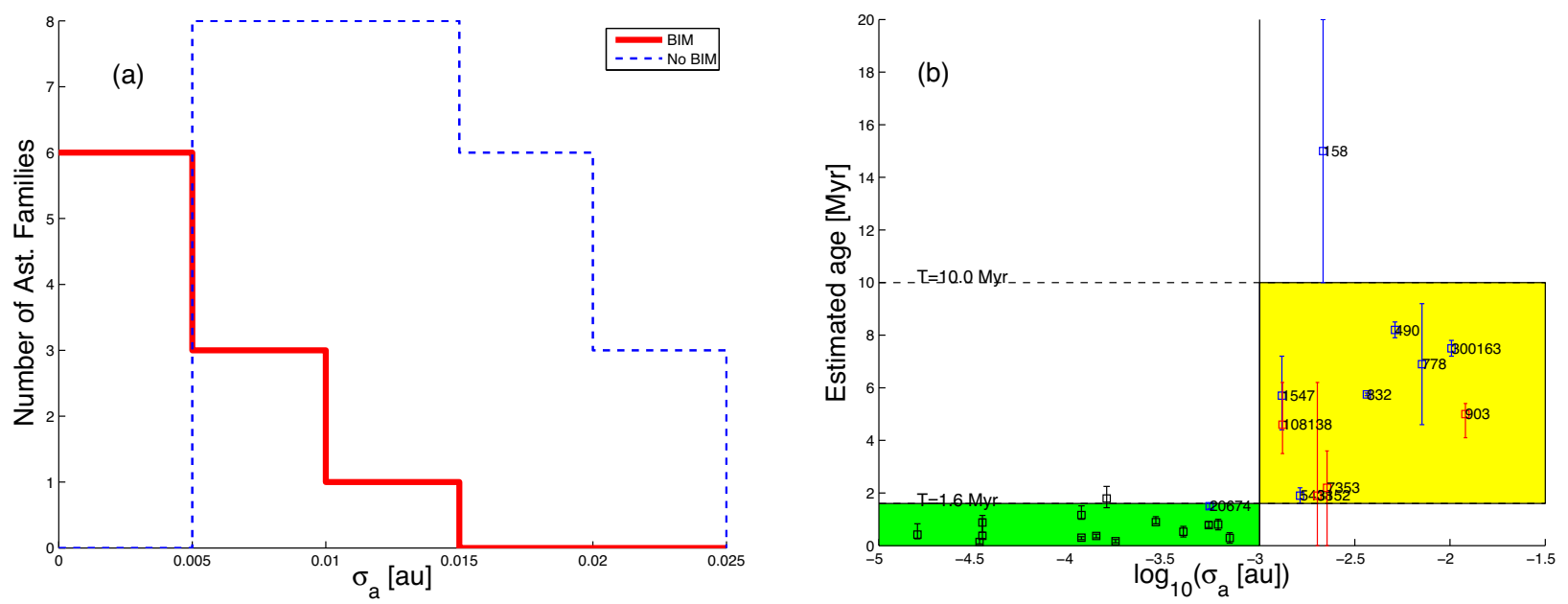

Figure 4. Panel (a): A histogram of the standard deviation in proper $a, \sigma_{a}$ for the 28 families studied in this work for which an age solution with BIM was (red line) or was not (blue dashed line) available. With the exception of (909) Ulla, most of the BIM families had values of $\sigma_{a}<0.0075$ [au]. Panel (b) displays the the BIM ages and their uncertainties for the families and clusters for which such information is available, as a function of their $\sigma_{a}$. Families identified in this work are displayed in red. The vertical line sets the approximate limit of $\sigma_{a}=0.001$ au that separates families identified in domain of proper elements from clusters identified in domains of osculating or mean elements. The horizontal lines display the approximate time limits for which a cluster can be identified in osculating/mean elements ( $T=1.6 \mathrm{Myr}$ ) and for which a solution including Yarkovsky drift can be reliably obtained ( $T=10.0 \mathrm{Myr})$. The green area is mostly populated by clusters that could have originated by a secondary fission process (Jacobson \& Scheeres 2011) or by a cratering collision onto a nearly critically rotating primary (Vokrouhlický et al. 2017). The yellow area is the region where most of the BIM asteroid families identified in domains of proper elements are currently found.

Table 3. The estimated ages for the four families for which we obtained a solution. The first and second columns display the family FIN and name, the third the value of the minimum of $\chi_{0}$, the fourth the percentage of asteroids whose angles converge at the estimated family age to within $\pm 80^{\circ}$, and the fifth column displays the estimated age at a $68.3 \%$ probability confidence level. The first two families were classified as type A in Section 2, while the last two were type B.

\begin{tabular}{ccccc}
\hline FIN & $\begin{array}{c}\text { Family } \\
\text { Name }\end{array}$ & $\begin{array}{c}\left(\chi_{0}^{2}\right)_{\min } \\
{\left[\mathrm{rad}^{2}\right]}\end{array}$ & $\begin{array}{c}\text { Percentage of } \\
\text { conv. ast. }\end{array}$ & $\begin{array}{c}\text { Estimated } \\
\text { age [Myr] }\end{array}$ \\
\hline 538 & (3152) Jones & 47.9 & $95.5 \%$ & $1.9_{-1.9}^{+4.3}$ \\
521 & (7353) Kazuya & 98.0 & $50.0 \%$ & $2.2_{-2.2}^{+1.4}$ \\
417 & $(108138)$ 2001 GB 11 & 12.5 & $88.9 \%$ & $4.6_{-1.1}^{+1.6}$ \\
903 & (909) Ulla & 24.1 & $91.3 \%$ & $5.0_{-0.9}^{+0.4}(?)$ \\
\hline
\end{tabular}

//hamilton.dm.unipi.it/astdys/index.php?pc=5 (Milani et al. 2014, 2016)). The alleged parent body of each family may also have drifted because of close encounters with massive asteroids and Ceres, and this may also alter the preliminary age estimates provided in this paper. Finally, the effects of local dynamics on the convergence of secular angles also need to be well understood. We consider these and other issues as important challenges for future study of the behavior of young asteroid families.

\section{ACKNOWLEDGEMENTS}

We thank the reviewer of this paper, Dr. Federica Spoto, for comments and suggestions that significantly helped to improve the quality of this paper. We are also grateful o Dr. David Vokrouhlický for discussions and comments on a revised version of this manuscript. We would like to thank the São Paulo State Science Foundation (FAPESP) that supported this work via the grants 16/04476-8 and 2013/15357-1, and the Brazilian National Research Council (CNPq, grant 312313/2014-4). We acknowledge the use of data from the As- teroid Dynamics Site (AstDys) (http://hamilton.dm.unipi.it/astdys, Knežević \& Milani (2003)). This publication makes use of data products from the Wide-field Infrared Survey Explorer (WISE) and Near-Earth Objects (NEOWISE), which are a joint project of the University of California, Los Angeles, and the Jet Propulsion Laboratory/California Institute of Technology, funded by the National Aeronautics and Space Administration.

\section{REFERENCES}

Brož M., 1999, Orbital Evolution of the Asteroid Fragments due to Planetary Perturbations and Yarkovsky Effects. Diploma Thesis, Charles University, Prague, Czech Republic

Brož M., Morbidelli A., Bottke W. F., Rozehnal J., Vokrouhlický D., Nesvorný D., 2013, A\&A, 551, A117

Carruba V., 2010. MNRAS, 408, 580

Carruba V., Nesvorný D., Aljbaae S., Huaman M. E., 2015, MNRAS, 451, 244

Carruba V., Nesvorný D., Vokrouhlický D., 2016. AJ, 151, 64.

Carruba V., Vokrouhlický D., Nesvorný D., 2017, MNRAS, 469, 4400.

Carruba V., Vokrouhlický D., Nesvorný D., Aljbaae S., 2018, MNRAS, 477, 1308.

Delbo M., dell'Oro A., Harris A. W., Mottola S., Mueller M., 2007, Icarus, 190, 236

Ďurech J., Carry B., Delbò M., Kaasalainen M., Viikinkoski M., 2015, in Michel P., DeMeo F. E., Bottke W., eds, Asteroids IV. University of Arizona Press, Tucson, p. 183

Ďurech J., Sidorin V., Kaasalainen M., 2010, A\&A, 513, A46

Jacobson S. A., Scheeres D. J., 2011, Icarus, 214, 161

Knežević Z., Milani A., 2003, A\&A, 403, 1165

Levison H. F., Duncan M. J., 1994, Icarus, 108, 18

Masiero J. R., Mainzer A. K., Bauer J. M. et al., 2013, ApJ, 770, 7

Milani A., Cellino A., Knežević Z. et al., 2014, Icarus, 239, 46

Milani A., Spoto F., Knežević Z., Novaković B., Tsirvoulis G., 2016, Proceedings of the International Astronomical Union, 10(S318) 28

Molnar L. A., Haegert M. J., 2009, AAS/Division for Planetary Sciences Meeting Abstracts \#41, 41, 27.05 
Nesvorný D., Bottke W. F., 2004, Icarus, 170, 324

Nesvorný D., Bottke W. F., Dones. L., Levison H. F., 2002, Nature, 417, 720

Nesvorný D., Bottke W. F., Levison H. F., Dones L., 2003, ApJ, 591, 486

Nesvorný D., Bottke W. F., Vokrouhlický D., Sykes M., Lien D. J., Stansberry J., 2008, AJ, L143

Nesvorný D., Brož M., Carruba V., 2015. in Michel P., DeMeo F. E., Bottke W., eds, Identification and Dynamical Properties of Asteroid Families, In Asteroid IV, The University of Arizona Press, p. 297

Nesvorný D., Vokrouhlický D., 2006, AJ, 132, 1950

Nesvorný D., Vokrouhlický D., Bottke W. F., 2006, Science, 312, 1490

Novaković B., 2010, MNRAS, 407, 1477

Novaković B., Dell'Oro A., Cellino A., Knežević Z., 2012, MNRAS, 425, 338

Novaković B., Hsieh H. H., Cellino A., 2012a, MNRAS, 424, 1432

Novaković B., Hsieh H. H., Cellino A., Micheli M., Pedani M., 2014, Icarus, 231, 300

Pravec P., Fatka P., Vokrouhlický D. et al., 2018, Icarus, 304, 110
Pravec P., Vokrouhlický D., 2009, Icarus, 204, 580

Radović V., 2017, MNRAS, 471, 1321

Rosaev A., Plávalová E., 2017, Icarus, 282, 326

Spoto F., Milani A., Knežević Z., 2015, Icarus, 257, 275

Tsiganis K., Knežević Z., Varvoglis H., 2007, Icarus, 186, 484

Vokrouhlický D., Bottke W. F., Chesley S. R., Scheeres D. J., Statler T. S., 2015. in Michel P., DeMeo F. E., Bottke W., eds, The Yarkovsky and YORP Effects, In Asteroid IV, The University of Arizona Press, p. 509 Vokrouhlický D., Durech J., Michałowski T. et al., 2009, A\&A, 507, 495

Vokrouhlický D., Nesvorný D., 2011, AJ, 142, 26

Vokrouhlický D., Pravec P., Durech J. et al., 2017, A\&A, 598, A91

Vokrouhlický D., Ďurech J., Pravec P. et al., 2016, AJ, 151, 56

This paper has been typeset from a $\mathrm{T}_{\mathrm{E}} \mathrm{X} / \mathrm{LAT}_{\mathrm{E}} \mathrm{X}$ file prepared by the author. 